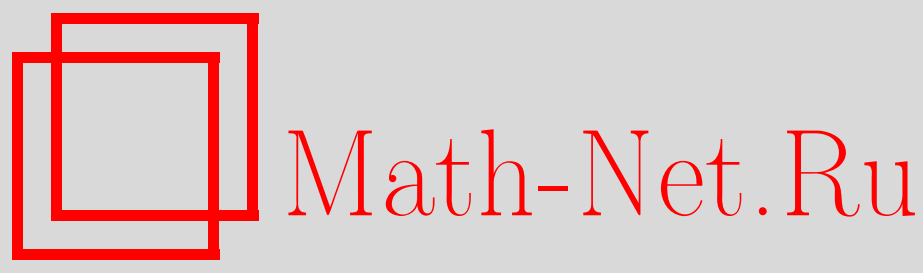

А. В. Одесский, В. В. Соколов, Интегрируемые эллиптические псевдопотенциалы, ТМФ, 2009, том 161, номер 1, 21-36

DOI: https://doi.org/10.4213/tmf6416

Использование Общероссийского математического портала Math-Net.Ru подразумевает, что вы прочитали и согласны с пользовательским соглашением http://www . mathnet.ru/rus/agreement

Параметры загрузки:

IP : 54.157 .27 .8

26 апреля 2023 г., $12: 47: 02$

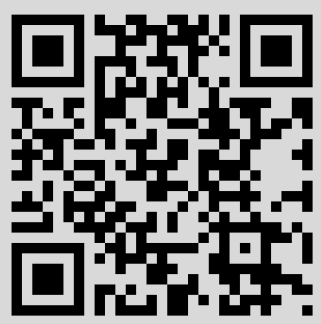




\title{
ФИЗИКА
}

Том 161, № 1

октябрь, 2009

2009 г.

\author{
А. В. Одесский*†, В. В. Соколов*
}

\section{ИНТЕГРИРУЕМЫЕ ЭЛЛИПТИЧЕСКИЕ ПСЕВДОПОТЕНЦИАЛЫ}

Интегрируемые псевдопотенциалы с произвольным числом полей построены в терминах эллиптического обобщения гипергеометрических функций нескольких аргументов. Эти псевдопотенциалы являются многопараметрическими деформациями псевдопотенциалов, построенных Кричевером при изучении усреднений по Уизему решений уравнения КП, и порождают новые интегрируемые $(2+1)$-мерные системы гидродинамического типа. Кроме того, с ними связан интересный класс интегрируемых $(1+1)$-мерных систем, описанный в терминах решений эллиптического обобщения системы Гиббонса-Царева.

Ключевые слова: интегрируемая трехмерная система гидродинамического типа, эллиптико-гипергеометрические функции.

\section{1. ВВЕДЕНИЕ}

В работе [1] был построен широкий класс квазилинейных интегрируемых систем уравнений в частных производных вида

$$
\sum_{j=1}^{m} a_{i j}(\mathbf{u}) u_{j, t_{1}}+\sum_{j=1}^{m} b_{i j}(\mathbf{u}) u_{j, t_{2}}+\sum_{j=1}^{m} c_{i j}(\mathbf{u}) u_{j, t_{3}}=0, \quad i=1, \ldots, l,
$$

где $\mathbf{u}=\left(u_{1}, \ldots, u_{m}\right)$. Коэффициенты этих уравнений были записаны в терминах обобщенных гипергеометрических функций [2]. Под интегрируемостью уравнения (1) мы понимаем существование псевдопотенциального представления ${ }^{1)}$

$$
\psi_{t_{2}}=A(p, \mathbf{u}), \quad \psi_{t_{3}}=B(p, \mathbf{u}), \quad \text { где } \quad p=\psi_{t_{1}} .
$$

Такое представление - это бездисперсионная версия [3], [4] представления нулевой кривизны, которое является основным понятием теории интегрируемых солитонных уравнений (см. статью [5]).

1)Это означает, что (1) эквивалентно условиям совместности для (2).

* Институт теоретической физики им. Л. Д. Ландау РАН, Москва, Россия. E-mail: sokolov@itp.ac.ru

${ }^{\dagger}$ Brock University, St. Catharines, Ontario, Canada. E-mail: aodesski@brocku.ca 
Одной из интересных и привлекательных черт теории интегрируемых систем (1) является тот факт, что зависимость псевдопотенциала от $p$ может быть гораздо более сложной, чем в солитонном случае. В работах [6], [7] были найдены важные примеры псевдопотенциалов $A, B$, связанных с процедурой усреднения по Уизему интегрируемых дисперсионных уравнений и с фробениусовыми многообразиями. Эти примеры ассоциированы с универсальной алгебраической кривой рода $g$ с $M$ проколами, где $g$ и $M$ произвольны, а коэффициенты кривой зависят от $\mathbf{u}$. Более точно, при всех $p \in \mathbb{C}$ точка $\left(A_{p p p} / A_{p p}^{2}, A_{p}\right)$ принадлежит кривой рода $g$, а $\mathbf{u}$ - это некоторые координаты на пространстве $\mathcal{M}_{g, M}$ модулей кривых рода $g$ с $M$ проколами.

Псевдопотенциалы из работы [1] (см. также [8]) были записаны в следующей параметрической форме: $A=F_{1}(\xi, \mathbf{u}), p=F_{2}(\xi, \mathbf{u})$, где зависимость функций $F_{i}$ от переменной $\xi$ определяется обыкновенным дифференциальным уравнением

$$
F_{i, \xi}=\phi_{i}(\xi, \mathbf{u}) \cdot \xi^{-s_{1}}(\xi-1)^{-s_{2}}\left(\xi-u_{1}\right)^{-s_{3}} \ldots\left(\xi-u_{m}\right)^{-s_{m+2}} .
$$

Здесь $s_{1}, \ldots, s_{m+2}$ - произвольные постоянные, а $\phi_{i}-$ многочлены степени $m-k$ по переменной $\xi$. Эти псевдопотенциалы связаны с рациональными алгебраическими кривыми. Если $s_{1}=\cdots=s_{m+2}=0$ и $k=0$, то они совпадают с псевдопотенциалами из работы [6], соответствующими $\mathcal{M}_{0, m+3}$.

В настоящей статье мы строим псевдопотенциалы и интегрируемые системы (1), связанные с эллиптической кривой. Для этих систем $\mathbf{u}=\left(u_{1}, \ldots, u_{n}, \tau\right)$, где $\tau-$ это параметр эллиптической кривой, который также является неизвестной функцией в (1). Коэффициенты систем выражаются в терминах некоторого эллиптического обобщения гипергеометрических функций нескольких переменных [9]. Эти эллиптико-гипергеометрические функции определяются как решения следующей линейной переопределенной совместной системы уравнений в частных производных:

$$
\begin{aligned}
g_{u_{\alpha} u_{\beta}}= & s_{\beta}\left(\rho\left(u_{\beta}-u_{\alpha}\right)+\rho\left(u_{\alpha}+\eta\right)-\rho\left(u_{\beta}\right)-\rho(\eta)\right) g_{u_{\alpha}}+ \\
& +s_{\alpha}\left(\rho\left(u_{\alpha}-u_{\beta}\right)+\rho\left(u_{\beta}+\eta\right)-\rho\left(u_{\alpha}\right)-\rho(\eta)\right) g_{u_{\beta}}, \\
g_{u_{\alpha} u_{\alpha}}= & s_{\alpha} \sum_{\beta \neq \alpha}\left(\rho\left(u_{\alpha}\right)+\rho(\eta)-\rho\left(u_{\alpha}-u_{\beta}\right)-\rho\left(u_{\beta}+\eta\right)\right) g_{u_{\beta}}+ \\
& +\left(\sum_{\beta \neq \alpha} s_{\beta} \rho\left(u_{\alpha}-u_{\beta}\right)+\left(s_{\alpha}+1\right) \rho\left(u_{\alpha}+\eta\right)+\right. \\
& \left.+s_{\alpha} \rho(-\eta)+\left(s_{0}-s_{\alpha}-1\right) \rho\left(u_{\alpha}\right)+2 \pi i r\right) g_{u_{\alpha}}-s_{0} s_{\alpha}\left(\rho^{\prime}\left(u_{\alpha}\right)-\rho^{\prime}(\eta)\right) g, \\
g_{\tau}= & \frac{1}{2 \pi i} \sum_{\beta}\left(\rho\left(u_{\beta}+\eta\right)-\rho(\eta)\right) g_{u_{\beta}}-\frac{s_{0}}{2 \pi i} \rho^{\prime}(\eta) g
\end{aligned}
$$

относительно одной функции $g\left(u_{1}, \ldots, u_{n}, \tau\right)$. Здесь $\eta=s_{1} u_{1}+\cdots+s_{n} u_{n}+r \tau+\eta_{0}$, $s_{0}=-s_{1}-\cdots-s_{n}$, где $s_{1}, \ldots, s_{n}, r, \eta_{0}-$ произвольные постоянные, а

$$
\theta(z)=\sum_{\alpha \in \mathbb{Z}}(-1)^{\alpha} e^{2 \pi i(\alpha z+\alpha(\alpha-1) \tau / 2)}, \quad \rho(z)=\frac{\theta^{\prime}(z)}{\theta(z)} .
$$


В формулах (4), а также всюду ниже, мы опускаем второй аргумент функций $\theta, \rho$ и используем обозначения

$$
\rho^{\prime}(z)=\frac{\partial \rho(z)}{\partial z}, \quad \rho_{\tau}(z)=\frac{\partial \rho(z)}{\partial \tau}, \quad \theta^{\prime}(z)=\frac{\partial \theta(z)}{\partial z}, \quad \theta_{\tau}(z)=\frac{\partial \theta(z)}{\partial \tau}
$$

Оказывается, что размерность пространства решений системы (4) равна $n+1$.

Статья имеет следующую структуру. В разделе 2 мы описываем некоторые полезные для наших целей свойства эллиптико-гипергеометрических функций. В частности, мы приводим интегральное представление, аналогичное представлению для обобщенных гипергеометрических функций (см., например, [1]).

В разделе 3 для любого $n$ мы строим псевдопотенциалы представления (2) с $k=0$, связанные с эллиптико-гипергеометрическими функциями. Эти псевдопотенциалы $A_{n}\left(p, u_{1}, \ldots, u_{n}, \tau\right)$ заданы параметрически:

$$
A_{n}=P_{n}\left(g_{1}, \xi\right), \quad p=P_{n}\left(g_{0}, \xi\right),
$$

где $g_{1}, g_{0}$ - линейно независимые решения системы (4),

$$
\begin{aligned}
& P_{n}(g, \xi)=\int_{0}^{\xi} S_{n}(g, \xi) e^{2 \pi i r(\tau-\xi)} \frac{\theta^{\prime}(0)^{-s_{1}-\cdots-s_{n}} \theta\left(u_{1}\right)^{s_{1}} \ldots \theta\left(u_{n}\right)^{s_{n}}}{\theta(\xi)^{-s_{1}-\cdots-s_{n}} \theta\left(\xi-u_{1}\right)^{s_{1}} \ldots \theta\left(\xi-u_{n}\right)^{s_{n}}} d \xi, \\
& S_{n}(g, \xi)=\sum_{1 \leqslant \alpha \leqslant n} \frac{\theta\left(u_{\alpha}\right) \theta\left(\xi-u_{\alpha}-\eta\right)}{\theta\left(u_{\alpha}+\eta\right) \theta\left(\xi-u_{\alpha}\right)} g_{u_{\alpha}}-\left(s_{1}+\cdots+s_{n}\right) \frac{\theta^{\prime}(0) \theta(\xi-\eta)}{\theta(\eta) \theta(\xi)} g .
\end{aligned}
$$

Псевдопотенциал $A_{n}\left(p, u_{1}, \ldots, u_{n}, \tau\right)$, определенный параметрически формулой (6), называется эллиптическим псевдопотенциалом дефекта ноль. Псевдопотенциалы $A_{n}\left(p, u_{1}, \ldots, u_{n}, \tau\right), n=1,2, \ldots$, определяют интегрируемые системы вида (1) с $m=l=n+1$. В случае $s_{1}=\cdots=s_{n}=r=0, \eta_{0} \rightarrow 0$ наши псевдопотенциалы совпадают с эллиптическими псевдопотенциалами, построенными в работе [6].

В разделе 4 для $k<n$ мы строим псевдопотенциалы дефекта $k$, которые определяют системы (1) с $m=n+1, l=n+k+1$. Специальный класс решений интегрируемых систем (1), зависящий от нескольких произвольных функций одной переменной, может быть построен методом гидродинамических редукций [10], [11]. Отметим, что в работе [11] существование гидродинамических редукций использовалось также в качестве эффективного критерия интегрируемости для систем (1). Гидродинамические редукции определяются парами интегрируемых совместных $(1+1)$-мерных систем гидродинамического типа

$$
r_{t}^{i}=v^{i}\left(r^{1}, \ldots, r^{N}\right) r_{x}^{i}, \quad i=1,2, \ldots, N
$$

Такие интегрируемые системы также интересны сами по себе. Общая теория этих систем была развита в работах [12], [13]. 
Раздел 5 посвящен гидродинамическим редукциям систем (1), построенных в разделах 3 и 4 . В случае $k=0$ соответствующие системы (9) имеют вид

$$
r_{t}^{i}=\frac{S_{n}\left(g_{1}(\mathbf{u}), \xi_{i}\right)}{S_{n}\left(g_{2}(\mathbf{u}), \xi_{i}\right)} r_{x}^{i},
$$

где $g_{1}, g_{2}$ - линейно независимые решения системы (4). Для потенциалов дефекта $k>0$ соответствующая формула аналогична. Функции $\tau\left(r^{1}, \ldots, r^{N}\right), \xi_{i}\left(r^{1}, \ldots, r^{N}\right)$, $u_{i}\left(r^{1}, \ldots, r^{N}\right)$ определяются следующей универсальной переопределенной совместной системой уравнений в частных производных типа системы Гиббонса-Царева [10], [14]:

$$
\begin{aligned}
\partial_{\alpha} \xi_{\beta} & =\frac{1}{2 \pi i}\left(\rho\left(\xi_{\alpha}-\xi_{\beta}\right)-\rho\left(\xi_{\alpha}\right)\right) \partial_{\alpha} \tau, \quad \partial_{\alpha}=\frac{\partial}{\partial r_{\alpha}} \\
\partial_{\alpha} \partial_{\beta} \tau & =-\frac{1}{\pi i} \rho^{\prime}\left(\xi_{\alpha}-\xi_{\beta}\right) \partial_{\alpha} \tau \partial_{\beta} \tau
\end{aligned}
$$

где $\alpha, \beta=1, \ldots, N, \alpha \neq \beta$, и

$$
\partial_{\alpha} u_{\beta}=\frac{1}{2 \pi i}\left(\rho\left(\xi_{\alpha}-u_{\beta}\right)-\rho\left(\xi_{\alpha}\right)\right) \partial_{\alpha} \tau, \quad \alpha=1, \ldots, N, \quad \beta=1, \ldots, n .
$$

Напомним, что здесь $\tau$ - это второй аргумент функции $\rho$. В другой связи похожие формулы появлялись в работе [15]. Легко проверить, что система (11)-(13) совместна и, следовательно, $(1+1)$-мерные системы (10) допускают локальную параметризацию с помощью $2 N$ произвольных функций одной переменной.

Для некоторых очень специальных значений параметров $s_{i}$ в (4) системы (10) связаны с уиземовскими иерархиями [6], с фробениусовыми многообразиями и с уравнением ассоциативности [7], [16].

\section{2. ЭЛЛИПТИКО-ГИПЕРГЕОМЕТРИЧЕСКИЕ ФУНКЦИИ}

Определим функцию $\theta$ двух переменных $z, \tau$ формулой (5), предполагая, что $\operatorname{Im} \tau>0$. Функция $\theta$ называется $\theta$-функцией порядка 1 от одной переменной. Напомним следующие полезные формулы:

$$
\begin{aligned}
\theta(z+1) & =\theta(z), & \theta(z+\tau) & =-e^{-2 \pi i z} \theta(z) \\
\theta(-z) & =-e^{-2 \pi i z} \theta(z), & \theta_{\tau}(z) & =\frac{1}{4 \pi i} \theta^{\prime \prime}(z)-\frac{1}{2} \theta^{\prime}(z) .
\end{aligned}
$$

Следующие утверждения проверяются непосредственно.

ПредлОЖЕНИЕ 1. Система линейнъх уравнений (4) совместна для любых постоянных $s_{1}, \ldots, s_{n}, r, \eta_{0}$. Размерность линейного пространства $\mathcal{H}$ решений системы (4) равна $n+1$. 
ЗАмЕЧАНИЕ 1. Коэффициенты в (4) могут быть записаны в факторизованной форме с помощью тождества

$$
\rho\left(u_{\beta}-u_{\alpha}\right)+\rho\left(u_{\alpha}+\eta\right)-\rho\left(u_{\beta}\right)-\rho(\eta)=-\frac{\theta^{\prime}(0) \theta\left(u_{\alpha}-u_{\beta}+\eta\right) \theta\left(u_{\alpha}\right) \theta\left(u_{\beta}+\eta\right)}{\theta\left(u_{\alpha}-u_{\beta}\right) \theta\left(u_{\alpha}+\eta\right) \theta\left(u_{\beta}\right) \theta(\eta)} .
$$

Назовем элементы пространства $\mathcal{H}$ эллиптико-гипергеометрическими функицями. В случае, когда нам важны значения параметров системы (4), будем обозначать $\mathcal{H}$ через $\mathcal{H}_{s_{1}, \ldots, s_{n}, r, \eta_{0}}$.

ПреДЛОЖЕНИЕ 2. Определим функиию $F\left(u_{1}, \ldots, u_{n}, \tau\right)$ с помощъю следующего интегрального представления:

$$
\begin{aligned}
F\left(u_{1}, \ldots, u_{n}, \tau\right)= & \int_{0}^{1} \frac{\theta\left(u_{1}-t\right)^{s_{1}} \ldots \theta\left(u_{n}-t\right)^{s_{n}} \theta^{\prime}(0)^{s_{1}+\cdots+s_{n}+1} \theta(t+\eta)}{\theta\left(u_{1}\right)^{s_{1}} \ldots \theta\left(u_{n}\right)^{s_{n}} \theta(t)^{s_{1}+\cdots+s_{n}+1} \theta(\eta)} \times \\
& \times e^{2 \pi i\left(s_{1}+\cdots+s_{n}+r\right) t} d t .
\end{aligned}
$$

Тогда F удовлетворяет системе (4).

ПрЕДЛОЖЕНИЕ 3. Пусть $\mathcal{H}=\mathcal{H}_{s_{1}, \ldots, s_{n}, r, \eta_{0}} u \widetilde{\mathcal{H}}=\mathcal{H}_{s_{1}, \ldots, s_{n}, 0, r, \eta_{0}}$. Тогда $\widetilde{\mathcal{H}}$ порождено элементами $\mathcal{H}$ и функиией

$$
\begin{aligned}
Z\left(u_{1}, \ldots, u_{n}, u_{n+1}, \tau\right)= & \int_{0}^{u_{n+1}} \frac{\theta\left(u_{1}-t\right)^{s_{1}} \ldots \theta\left(u_{n}-t\right)^{s_{n}} \theta^{\prime}(0)^{s_{1}+\cdots+s_{n}+1} \theta(t+\eta)}{\theta\left(u_{1}\right)^{s_{1}} \ldots \theta\left(u_{n}\right)^{s_{n}} \theta(t)^{s_{1}+\cdots+s_{n}+1} \theta(\eta)} \times \\
& \times e^{2 \pi i\left(s_{1}+\cdots+s_{n}+r\right) t} d t
\end{aligned}
$$

Более того, пространство $\mathcal{H}_{s_{1}, \ldots, s_{n}, 0, \ldots, 0, r, \eta_{0}}$ (среди параметров $m$ нулей) порождено $\mathcal{H}$ и $Z\left(u_{1}, \ldots, u_{n}, u_{n+1}, \tau\right), Z\left(u_{1}, \ldots, u_{n}, u_{n+2}, \tau\right), \ldots, Z\left(u_{1}, \ldots, u_{n}, u_{n+m}, \tau\right)$.

В простейшем случае $n=1$ система (4) относительно функции $g\left(u_{1}, \tau\right)$ имеет следующий вид:

$$
\begin{aligned}
g_{u_{1} u_{1}}= & \left(\left(s_{1}+1\right) \frac{\theta^{\prime}\left(u_{1}+\eta\right)}{\theta\left(u_{1}+\eta\right)}+s_{1} \frac{\theta^{\prime}(-\eta)}{\theta(-\eta)}-\left(2 s_{1}+1\right) \frac{\theta^{\prime}\left(u_{1}\right)}{\theta\left(u_{1}\right)}+2 \pi i r\right) g_{u_{1}}- \\
& -\frac{s_{1}^{2} \theta^{\prime}(0)^{2} \theta\left(u_{1}-\eta\right) \theta\left(u_{1}+\eta\right)}{\theta\left(u_{1}\right)^{2} \theta(-\eta) \theta(\eta)} g, \\
g_{\tau}= & \frac{1}{2 \pi i}\left(\frac{\theta^{\prime}\left(u_{1}+\eta\right)}{\theta\left(u_{1}+\eta\right)}-\frac{\theta^{\prime}(\eta)}{\theta(\eta)}\right) g_{u_{1}}+\frac{s_{1}}{2 \pi i}(\ln \theta(\eta))^{\prime \prime} g .
\end{aligned}
$$


Если $s_{1}=0$, то функции $g=1$ и

$$
g=Z\left(u_{1}, \tau\right)=\int_{0}^{u_{1}} \frac{\theta^{\prime}(0) \theta(t+\eta)}{\theta(t) \theta(\eta)} e^{2 \pi i r t} d t
$$

порождают пространство решений. Из предложения 3 следует, что функции 1 , $Z\left(u_{1}, \tau\right), \ldots, Z\left(u_{n}, \tau\right)$ порождают пространство решений системы (4) в случае $s_{1}=\cdots=s_{n}=0$.

\section{3. ЭЛЛИПТИЧЕСКИЕ ПСЕВДОПОТЕНЦИАЛЫ ДЕФЕКТА НОЛЬ}

Для любой эллиптико-гипергеометрической функции $g \in \mathcal{H}$ определим $P_{n}(g, \xi)$ формулами $(7),(8)$ при $\operatorname{Re}\left(s_{1}+\cdots+s_{n}\right)>1$ и как аналитическое продолжение выражения (7) в противном случае.

Пусть $g_{1}, g_{0}$ - линейно независимые элементы $\mathcal{H}$. Соотношения (6) означают, что для нахождения эллиптического псевдопотенциала дефекта ноль $A_{n}\left(p, u_{1}, \ldots, u_{n}, \tau\right)$ нужно выразить $\xi$ из второго уравнения и подставить результат в первое уравнение.

Пусть $g_{0}, g_{1}, \ldots, g_{n}$ - базис в $\mathcal{H}$. Определим псевдопотенциалы $B_{\alpha}\left(p, u_{1}, \ldots, u_{n}, \tau\right)$ дефекта ноль формулой

$$
B_{\alpha}=P_{n}\left(g_{\alpha}, \xi\right), \quad p=P_{n}\left(g_{0}, \xi\right), \quad \alpha=1, \ldots, n
$$

Предположим, что $u_{1}, \ldots, u_{n}, \tau$ являются функциями от $t_{0}=x, t_{1}, \ldots, t_{n}$.

ТЕорема 1. Условия совместности $\psi_{t_{\alpha} t_{\beta}}=\psi_{t_{\beta} t_{\alpha}}$ для системь

$$
\psi_{t_{\alpha}}=B_{\alpha}\left(\psi_{x}, u_{1}, \ldots, u_{n}, \tau\right), \quad \alpha=1, \ldots, n
$$

эквивалентны следующей системе уравнений в частных производных относительно функиий $u_{1}, \ldots, u_{n}, \tau$ :

$$
\begin{aligned}
& \sum_{1 \leqslant \beta \leqslant n}\left(g_{q} g_{r, u_{\beta}}-g_{r} g_{q, u_{\beta}}\right)\left(u_{\beta, t_{s}}+\frac{1}{2 \pi i}\left(\frac{\theta^{\prime}\left(u_{\beta}+\eta\right)}{\theta\left(u_{\beta}+\eta\right)}-\frac{\theta^{\prime}(\eta)}{\theta(\eta)}\right) \tau_{t_{s}}\right)+ \\
& \quad+\sum_{1 \leqslant \beta \leqslant n}\left(g_{r} g_{s, u_{\beta}}-g_{s} g_{r, u_{\beta}}\right)\left(u_{\beta, t_{q}}+\frac{1}{2 \pi i}\left(\frac{\theta^{\prime}\left(u_{\beta}+\eta\right)}{\theta\left(u_{\beta}+\eta\right)}-\frac{\theta^{\prime}(\eta)}{\theta(\eta)}\right) \tau_{t_{q}}\right)+ \\
& \quad+\sum_{1 \leqslant \beta \leqslant n}\left(g_{s} g_{q, u_{\beta}}-g_{q} g_{s, u_{\beta}}\right)\left(u_{\beta, t_{r}}+\frac{1}{2 \pi i}\left(\frac{\theta^{\prime}\left(u_{\beta}+\eta\right)}{\theta\left(u_{\beta}+\eta\right)}-\frac{\theta^{\prime}(\eta)}{\theta(\eta)}\right) \tau_{t_{r}}\right)=0
\end{aligned}
$$




$$
\begin{aligned}
& \sum_{\substack{1 \leqslant \beta \leqslant n, \beta \neq \alpha}} \frac{\theta\left(u_{\beta}\right) \theta\left(u_{\alpha}-u_{\beta}-\eta\right)}{\theta\left(u_{\beta}+\eta\right) \theta\left(u_{\alpha}-u_{\beta}\right)}\left(g_{r, u_{\alpha}} g_{q, u_{\beta}}-g_{q, u_{\alpha}} g_{r, u_{\beta}}\right) \times \\
& \times\left(u_{\alpha, t_{s}}-u_{\beta, t_{s}}+\frac{1}{2 \pi i}\left(\frac{\theta^{\prime}\left(u_{\alpha}-u_{\beta}-\eta\right)}{\theta\left(u_{\alpha}-u_{\beta}-\eta\right)}-\frac{\theta^{\prime}(-\eta)}{\theta(-\eta)}\right) \tau_{t_{s}}\right)+ \\
& +\sum_{\substack{1 \leqslant \beta \leqslant n \\
\beta \neq \alpha}} \frac{\theta\left(u_{\beta}\right) \theta\left(u_{\alpha}-u_{\beta}-\eta\right)}{\theta\left(u_{\beta}+\eta\right) \theta\left(u_{\alpha}-u_{\beta}\right)}\left(g_{s, u_{\alpha}} g_{r, u_{\beta}}-g_{r, u_{\alpha}} g_{s, u_{\beta}}\right) \times \\
& \times\left(u_{\alpha, t_{q}}-u_{\beta, t_{q}}+\frac{1}{2 \pi i}\left(\frac{\theta^{\prime}\left(u_{\alpha}-u_{\beta}-\eta\right)}{\theta\left(u_{\alpha}-u_{\beta}-\eta\right)}-\frac{\theta^{\prime}(-\eta)}{\theta(-\eta)}\right) \tau_{t_{q}}\right)+ \\
& +\sum_{\substack{1 \leqslant \beta \leqslant n \\
\beta \neq \alpha}} \frac{\theta\left(u_{\beta}\right) \theta\left(u_{\alpha}-u_{\beta}-\eta\right)}{\theta\left(u_{\beta}+\eta\right) \theta\left(u_{\alpha}-u_{\beta}\right)}\left(g_{q, u_{\alpha}} g_{s, u_{\beta}}-g_{s, u_{\alpha}} g_{q, u_{\beta}}\right) \times \\
& \times\left(u_{\alpha, t_{r}}-u_{\beta, t_{r}}+\frac{1}{2 \pi i}\left(\frac{\theta^{\prime}\left(u_{\alpha}-u_{\beta}-\eta\right)}{\theta\left(u_{\alpha}-u_{\beta}-\eta\right)}-\frac{\theta^{\prime}(-\eta)}{\theta(-\eta)}\right) \tau_{t_{r}}\right)- \\
& -\left(s_{1}+\cdots+s_{n}\right) \frac{\theta^{\prime}(0) \theta\left(u_{\alpha}-\eta\right)}{\theta(\eta) \theta\left(u_{\alpha}\right)}\left(g_{q} g_{r, u_{\alpha}}-g_{r} g_{q, u_{\alpha}}\right) \times \\
& \times\left(u_{\alpha, t_{s}}+\frac{1}{2 \pi i}\left(\frac{\theta^{\prime}\left(u_{\alpha}-\eta\right)}{\theta\left(u_{\alpha}-\eta\right)}-\frac{\theta^{\prime}(-\eta)}{\theta(-\eta)}\right) \tau_{t_{s}}\right)- \\
& -\left(s_{1}+\cdots+s_{n}\right) \frac{\theta^{\prime}(0) \theta\left(u_{\alpha}-\eta\right)}{\theta(\eta) \theta\left(u_{\alpha}\right)}\left(g_{r} g_{s, u_{\alpha}}-g_{s} g_{r, u_{\alpha}}\right) \times \\
& \times\left(u_{\alpha, t_{q}}+\frac{1}{2 \pi i}\left(\frac{\theta^{\prime}\left(u_{\alpha}-\eta\right)}{\theta\left(u_{\alpha}-\eta\right)}-\frac{\theta^{\prime}(-\eta)}{\theta(-\eta)}\right) \tau_{t_{q}}\right)- \\
& -\left(s_{1}+\cdots+s_{n}\right) \frac{\theta^{\prime}(0) \theta\left(u_{\alpha}-\eta\right)}{\theta(\eta) \theta\left(u_{\alpha}\right)}\left(g_{s} g_{q, u_{\alpha}}-g_{q} g_{s, u_{\alpha}}\right) \times \\
& \times\left(u_{\alpha, t_{r}}+\frac{1}{2 \pi i}\left(\frac{\theta^{\prime}\left(u_{\alpha}-\eta\right)}{\theta\left(u_{\alpha}-\eta\right)}-\frac{\theta^{\prime}(-\eta)}{\theta(-\eta)}\right) \tau_{t_{r}}\right)=0, \quad \alpha=1, \ldots, n .
\end{aligned}
$$

Здесъ $q, r, s$ меняются от 0 до $n$.

ЗАмЕчАниЕ 2. При фиксированных $t_{1}, t_{2}, t_{3}$ теорема 1 дает систему вида (1) с $l=m=n+1$, обладающую псевдопотенциальным представлением.

ЗАмечание 3 . Рассмотрим случай $s_{1}=\cdots=s_{n}=0$. Согласно результатам раздела 2 имеем

$$
g=c_{0}+c_{1} Z\left(u_{1}, \tau\right)+\cdots+c_{n} Z\left(u_{n}, \tau\right),
$$

где $c_{0}, \ldots, c_{n}-$ произвольные постоянные. Поэтому

$$
S_{n}(g, \xi)=\sum_{1 \leqslant \alpha \leqslant n} c_{\alpha} e^{2 \pi i r u_{\alpha}} \frac{\theta^{\prime}(0) \theta\left(\xi-u_{\alpha}-\eta_{0}\right)}{\theta\left(\eta_{0}\right) \theta\left(\xi-u_{\alpha}\right)} .
$$


Если предположить, что $r=0$ и $c_{1}+\cdots+c_{n}=0$, то в пределе $\eta_{0} \rightarrow 0$ получим

$$
S_{n}(g, \xi)=\sum_{1 \leqslant \alpha \leqslant n} c_{\alpha} \rho\left(\xi-u_{\alpha}\right)
$$

Система уравнений в частных производных, эквивалентная условиям совместности для уравнений вида (16), была названа в работе [6] иерархией Уизема. В этой статье были построены некоторые иерархии Уизема, связанные с алгебраическими кривыми произвольного рода $g$. Иерархия, соответствующая $g=1$, эквивалентна описанной в теореме 1 , если $r=s_{1}=\cdots=s_{n}=0, c_{1}+\cdots+c_{n}=0$ и $\eta_{0}$ стремится к нулю, как описано выше.

\section{4. ЭЛЛИПТИЧЕСКИЕ ПСЕВДОПОТЕНЦИАЛЫ ДЕФЕКТА $k>0$}

Построим эллиптические псевдопотенциалы дефекта $k$. Зафиксируем $k$ линейно независимых эллиптико-гипергеометрических функций $h_{1}, \ldots, h_{k} \in \mathcal{H}$. Для любой функции $g \in \mathcal{H}$ определим $P_{n, k}(g, \xi)$ формулой

$$
P_{n, k}(g, \xi)=\frac{1}{\Delta} \operatorname{det}\left(\begin{array}{cccc}
P_{n}(g, \xi) & P_{n}\left(h_{1}, \xi\right) & \ldots & P_{n}\left(h_{k}, \xi\right) \\
g_{u_{n-k+1}} & h_{1, u_{n-k+1}} & \ldots & h_{k, u_{n-k+1}} \\
\vdots & \vdots & \ddots & \vdots \\
g_{u_{n}} & h_{1, u_{n}} & \ldots & h_{k, u_{n}}
\end{array}\right) .
$$

Здесь

$$
\Delta=\operatorname{det}\left(\begin{array}{ccc}
h_{1, u_{n-k+1}} & \ldots & h_{k, u_{n-k+1}} \\
\vdots & \ddots & \vdots \\
h_{1, u_{n}} & \ldots & h_{k, u_{n}}
\end{array}\right)
$$

а $P_{n}(g, \xi)$ задано формулой (7). Отметим, что $P_{n, k}\left(h_{1}, \xi\right)=\cdots=P_{n, k}\left(h_{k}, \xi\right)=0$. Легко видеть, что линейные преобразования вида $h_{i} \rightarrow c_{i 1} h_{1}+\cdots+c_{i k} h_{k}, g \rightarrow$ $g+d_{1} h_{1}+\cdots+d_{k} h_{k}$ с постоянными коэффициентами $c_{i j}, d_{i}$ не меняют $P_{n, k}(g, \xi)$.

Можно проверить, что

$$
\left(P_{n, k}(g, \xi)\right)_{\xi}=S_{n, k}(g, \xi) e^{2 \pi i r(\tau-\xi)} \frac{\theta^{\prime}(0)^{-s_{1}-\cdots-s_{n}} \theta\left(u_{1}\right)^{s_{1}} \ldots \theta\left(u_{n}\right)^{s_{n}}}{\theta(\xi)^{-s_{1}-\cdots-s_{n}} \theta\left(\xi-u_{1}\right)^{s_{1}} \ldots \theta\left(\xi-u_{n}\right)^{s_{n}}},
$$

где

$$
\begin{array}{r}
S_{n, k}(g, \xi)=\frac{1}{\Delta}\left(\sum_{1 \leqslant \alpha \leqslant n-k} \frac{\theta\left(u_{\alpha}\right) \theta\left(\xi-u_{\alpha}-\eta\right)}{\theta\left(u_{\alpha}+\eta\right) \theta\left(\xi-u_{\alpha}\right)} \Delta_{\alpha}(g)-\right. \\
\left.-\left(s_{1}+\cdots+s_{n}\right) \frac{\theta^{\prime}(0) \theta(\xi-\eta)}{\theta(\eta) \theta(\xi)} \Delta_{0}(g)\right)
\end{array}
$$




$$
\begin{gathered}
\Delta_{\alpha}(g)=\operatorname{det}\left(\begin{array}{cccc}
g_{u_{\alpha}} & h_{1, u_{\alpha}} & \ldots & h_{k, u_{\alpha}} \\
g_{u_{n-k+1}} & h_{1, u_{n-k+1}} & \ldots & h_{k, u_{n-k+1}} \\
\vdots & \vdots & \ddots & \vdots \\
g_{u_{n}} & h_{1, u_{n}} & \ldots & h_{k, u_{n}}
\end{array}\right), \\
\Delta_{0}(g)=\operatorname{det}\left(\begin{array}{cccc}
g & h_{1} & \ldots & h_{k} \\
g_{u_{n-k+1}} & h_{1, u_{n-k+1}} & \ldots & h_{k, u_{n-k+1}} \\
\vdots & \vdots & \ddots & \vdots \\
g_{u_{n}} & h_{1, u_{n}} & \ldots & h_{k, u_{n}}
\end{array}\right) .
\end{gathered}
$$

Пусть $g_{1}, g_{2} \in \mathcal{H}$. Предположим, что $g_{1}, g_{2}, h_{1}, \ldots, h_{k}$ линейно независимы. Псевдопотенциал $A_{n, k}\left(p, u_{1}, \ldots, u_{n}, \tau\right)$, определенный параметрически формулой

$$
A_{n, k}=P_{n, k}\left(g_{1}, \xi\right), \quad p=P_{n, k}\left(g_{2}, \xi\right),
$$

называется эллиптическим псевдопотенциалом дефекта $k$.

Теорема 2. Пусть $g_{0}, g_{1}, \ldots, g_{n-k}, h_{1}, \ldots, h_{k}$ - базис в $\mathcal{H}$, a псевдопотенииалъ $B_{\alpha}$ определенъ формулами

$$
B_{\alpha}=P_{n, k}\left(g_{\alpha}, \xi\right), \quad p=P_{n, k}\left(g_{0}, \xi\right), \quad \alpha=1, \ldots, n-k
$$

Тогда условия совместности для системы (16) эквивалентны следующей системе уравнений в частных производных относительно $u_{1}, \ldots, u_{n}, \tau$ :

$$
\begin{aligned}
& \sum_{1 \leqslant \beta \leqslant n-k}\left(\Delta_{0}\left(g_{q}\right) \Delta_{\beta}\left(g_{r}\right)-\Delta_{0}\left(g_{r}\right) \Delta_{\beta}\left(g_{q}\right)\right)\left(u_{\beta, t_{s}}+\frac{1}{2 \pi i}\left(\frac{\theta^{\prime}\left(u_{\beta}+\eta\right)}{\theta\left(u_{\beta}+\eta\right)}-\frac{\theta^{\prime}(\eta)}{\theta(\eta)}\right) \tau_{t_{s}}\right)+ \\
& \quad+\sum_{1 \leqslant \beta \leqslant n-k}\left(\Delta_{0}\left(g_{r}\right) \Delta_{\beta}\left(g_{s}\right)-\Delta_{0}\left(g_{s}\right) \Delta_{\beta}\left(g_{r}\right)\right) \times \\
& \quad \times\left(u_{\beta, t_{q}}+\frac{1}{2 \pi i}\left(\frac{\theta^{\prime}\left(u_{\beta}+\eta\right)}{\theta\left(u_{\beta}+\eta\right)}-\frac{\theta^{\prime}(\eta)}{\theta(\eta)}\right) \tau_{t_{q}}\right)+ \\
& \quad+\sum_{1 \leqslant \beta \leqslant n-k}\left(\Delta_{0}\left(g_{s}\right) \Delta_{\beta}\left(g_{q}\right)-\Delta_{0}\left(g_{q}\right) \Delta_{\beta}\left(g_{s}\right)\right) \times \\
& \quad \times\left(u_{\beta, t_{r}}+\frac{1}{2 \pi i}\left(\frac{\theta^{\prime}\left(u_{\beta}+\eta\right)}{\theta\left(u_{\beta}+\eta\right)}-\frac{\theta^{\prime}(\eta)}{\theta(\eta)}\right) \tau_{t_{r}}\right)=0
\end{aligned}
$$




$$
\begin{aligned}
& \sum_{\substack{1 \leqslant \beta \leqslant n-k, \beta \neq \alpha}} \frac{\theta\left(u_{\beta}\right) \theta\left(u_{\alpha}-u_{\beta}-\eta\right)}{\theta\left(u_{\beta}+\eta\right) \theta\left(u_{\alpha}-u_{\beta}\right)}\left(\Delta_{\alpha}\left(g_{r}\right) \Delta_{\beta}\left(g_{q}\right)-\Delta_{\alpha}\left(g_{q}\right) \Delta_{\beta}\left(g_{r}\right)\right) \times \\
& \times\left(u_{\alpha, t_{s}}-u_{\beta, t_{s}}+\frac{1}{2 \pi i}\left(\frac{\theta^{\prime}\left(u_{\alpha}-u_{\beta}-\eta\right)}{\theta\left(u_{\alpha}-u_{\beta}-\eta\right)}-\frac{\theta^{\prime}(-\eta)}{\theta(-\eta)}\right) \tau_{t_{s}}\right)+ \\
& +\sum_{\substack{1 \leqslant \beta \leqslant n-k, \beta \neq \alpha}} \frac{\theta\left(u_{\beta}\right) \theta\left(u_{\alpha}-u_{\beta}-\eta\right)}{\theta\left(u_{\beta}+\eta\right) \theta\left(u_{\alpha}-u_{\beta}\right)}\left(\Delta_{\alpha}\left(g_{s}\right) \Delta_{\beta}\left(g_{r}\right)-\Delta_{\alpha}\left(g_{r}\right) \Delta_{\beta}\left(g_{s}\right)\right) \times \\
& \times\left(u_{\alpha, t_{q}}-u_{\beta, t_{q}}+\frac{1}{2 \pi i}\left(\frac{\theta^{\prime}\left(u_{\alpha}-u_{\beta}-\eta\right)}{\theta\left(u_{\alpha}-u_{\beta}-\eta\right)}-\frac{\theta^{\prime}(-\eta)}{\theta(-\eta)}\right) \tau_{t_{q}}\right)+ \\
& +\sum_{\substack{1 \leqslant \beta \leqslant n-k, \beta \neq \alpha}} \frac{\theta\left(u_{\beta}\right) \theta\left(u_{\alpha}-u_{\beta}-\eta\right)}{\theta\left(u_{\beta}+\eta\right) \theta\left(u_{\alpha}-u_{\beta}\right)}\left(\Delta_{\alpha}\left(g_{q}\right) \Delta_{\beta}\left(g_{s}\right)-\Delta_{\alpha}\left(g_{s}\right) \Delta_{\beta}\left(g_{q}\right)\right) \times \\
& \times\left(u_{\alpha, t_{r}}-u_{\beta, t_{r}}+\frac{1}{2 \pi i}\left(\frac{\theta^{\prime}\left(u_{\alpha}-u_{\beta}-\eta\right)}{\theta\left(u_{\alpha}-u_{\beta}-\eta\right)}-\frac{\theta^{\prime}(-\eta)}{\theta(-\eta)}\right) \tau_{t_{r}}\right)- \\
& -\left(s_{1}+\cdots+s_{n}\right) \frac{\theta^{\prime}(0) \theta\left(u_{\alpha}-\eta\right)}{\theta(\eta) \theta\left(u_{\alpha}\right)}\left(\Delta_{0}\left(g_{q}\right) \Delta_{\alpha}\left(g_{r}\right)-\Delta_{0}\left(g_{r}\right) \Delta_{\alpha}\left(g_{q}\right)\right) \times \\
& \times\left(u_{\alpha, t_{s}}+\frac{1}{2 \pi i}\left(\frac{\theta^{\prime}\left(u_{\alpha}-\eta\right)}{\theta\left(u_{\alpha}-\eta\right)}-\frac{\theta^{\prime}(-\eta)}{\theta(-\eta)}\right) \tau_{t_{s}}\right)- \\
& -\left(s_{1}+\cdots+s_{n}\right) \frac{\theta^{\prime}(0) \theta\left(u_{\alpha}-\eta\right)}{\theta(\eta) \theta\left(u_{\alpha}\right)}\left(\Delta_{0}\left(g_{r}\right) \Delta_{\alpha}\left(g_{s}\right)-\Delta_{0}\left(g_{s}\right) \Delta_{\alpha}\left(g_{r}\right)\right) \times \\
& \times\left(u_{\alpha, t_{q}}+\frac{1}{2 \pi i}\left(\frac{\theta^{\prime}\left(u_{\alpha}-\eta\right)}{\theta\left(u_{\alpha}-\eta\right)}-\frac{\theta^{\prime}(-\eta)}{\theta(-\eta)}\right) \tau_{t_{q}}\right)- \\
& -\left(s_{1}+\cdots+s_{n}\right) \frac{\theta^{\prime}(0) \theta\left(u_{\alpha}-\eta\right)}{\theta(\eta) \theta\left(u_{\alpha}\right)}\left(\Delta_{0}\left(g_{s}\right) \Delta_{\alpha}\left(g_{q}\right)-\Delta_{0}\left(g_{q}\right) \Delta_{\alpha}\left(g_{s}\right)\right) \times \\
& \times\left(u_{\alpha, t_{r}}+\frac{1}{2 \pi i}\left(\frac{\theta^{\prime}\left(u_{\alpha}-\eta\right)}{\theta\left(u_{\alpha}-\eta\right)}-\frac{\theta^{\prime}(-\eta)}{\theta(-\eta)}\right) \tau_{t_{r}}\right)=0, \quad \alpha=1, \ldots, n-k,
\end{aligned}
$$

$u$

$$
\begin{aligned}
\sum_{\alpha=1}^{n-k} & \frac{\Delta_{\alpha}\left(g_{r}\right) \theta\left(u_{\alpha}\right) \theta\left(u_{\beta}-u_{\alpha}-\eta\right)}{\Delta \theta\left(u_{\alpha}+\eta\right) \theta\left(u_{\beta}-u_{\alpha}\right)} u_{\alpha, t_{s}}-S_{n, k}\left(g_{r}, u_{\beta}\right) u_{\beta, t_{s}}- \\
& -\frac{1}{2 \pi i}\left(S_{n, k}^{\prime}\left(g_{r}, u_{\beta}\right)-\frac{\theta^{\prime}(-\eta)}{\theta(-\eta)} S_{n, k}\left(g_{r}, u_{\beta}\right)\right) \tau_{t_{s}}= \\
= & \sum_{\alpha=1}^{n-k} \frac{\Delta_{\alpha}\left(g_{s}\right) \theta\left(u_{\alpha}\right) \theta\left(u_{\beta}-u_{\alpha}-\eta\right)}{\Delta \theta\left(u_{\alpha}+\eta\right) \theta\left(u_{\beta}-u_{\alpha}\right)} u_{\alpha, t_{r}}-S_{n, k}\left(g_{s}, u_{\beta}\right) u_{\beta, t_{r}}- \\
& -\frac{1}{2 \pi i}\left(S_{n, k}^{\prime}\left(g_{s}, u_{\beta}\right)-\frac{\theta^{\prime}(-\eta)}{\theta(-\eta)} S_{n, k}\left(g_{s}, u_{\beta}\right)\right) \tau_{t_{r}}, \quad \beta=n-k+1, \ldots, n .
\end{aligned}
$$

Здесъ $q, r, s$ меняются от 0 до $n u t_{0}=x$. 
ЗАмЕчАниЕ 4 . При заданных $t_{1}, t_{2}, t_{3}$ теорема 2 приводит к системе вида (1) с $m=n+1, l=n+k+1$, обладающей псевдопотенциальным представлением. Действительно, формула (25) при $q, r, s=1,2,3$ дает $3 k$ линейно независимых уравнений. Формулы (23), (24) дают $n-k+1$ уравнений. С другой стороны, можно построить ровно $k$ линейных комбинаций уравнений (25) с $q, r, s=1,2,3$ таких, что производные функции $u_{i}, i=n-k+1, \ldots, n$, сокращаются. Оказывается, что эти линейные комбинации линейно зависимы с уравнениями $(23),(24)$. Поэтому существует $(n-k+1)+3 k-k=n+k+1$ линейно независимых уравнений.

ЗАмечАниЕ 5. Мы должны считать, что в (23)-(25) $n \geqslant k+2$. Действительно, для $n=k+1$ мы не можем построить более чем один псевдопотенциал, поэтому не существует $(2+1)$-мерной системы $(1)$, связанной с этим случаем. Тем не менее, соответствующий псевдопотенциал порождает интересные интегрируемые $(1+1)$-мерные системы гидродинамического типа (см. раздел 5). Вероятно, эти псевдопотенциалы для $k=0,1, \ldots$ также связаны с некоторыми бесконечными цепочками типа цепочки Бенни [17], [18].

Система (23)-(25) обладает законами сохранения гидродинамического типа. В частности, следующее утверждение может быть проверено непосредственно.

ПреДЛОЖЕНИЕ 4. Для любъх $r, s=0,1, \ldots, n, r \neq s$, система (23)-(25) имеет $k$ законов сохранения вида

$$
\left(\frac{\Delta\left(g_{r}, h_{1}, \ldots \hat{i} \ldots, h_{k}\right)}{\Delta\left(h_{1}, \ldots, h_{k}\right)}\right)_{t_{s}}=\left(\frac{\Delta\left(g_{s}, h_{1}, \ldots \hat{i} \ldots, h_{k}\right)}{\Delta\left(h_{1}, \ldots, h_{k}\right)}\right)_{t_{r}},
$$

где $i=1, \ldots, k$, а символ $\hat{i}$ означает отсутствие среди аргументов элемента $h_{i}$. Здесь

$$
\Delta\left(f_{1}, \ldots, f_{k}\right)=\operatorname{det}\left(\begin{array}{ccc}
f_{1, u_{n-k+1}} & \cdots & f_{k, u_{n-k+1}} \\
\vdots & \ddots & \vdots \\
f_{1, u_{n}} & \cdots & f_{k, u_{n}}
\end{array}\right) .
$$

Предложение 4 позволяет определить функции $z_{1}, \ldots, z_{k}$ такие, что

$$
\frac{\Delta\left(g_{r}, h_{1}, \ldots \hat{i} \ldots, h_{k}\right)}{\Delta\left(h_{1}, \ldots, h_{k}\right)}=z_{i, t_{r}}, \quad i=1, \ldots, k, \quad r=0,1, \ldots, n .
$$

Предположим, что $n+1 \geqslant 3 k$. Тогда система (1), полученная из системы (23)-(25) с $q, r, s=1,2,3$, состоит из $3 k$ уравнений $(25)$ (они эквивалентны уравнениям (26)) и $n+1-2 k$ уравнений $(23),(24)$. Действительно, только $n+1-2 k$ уравнений $(23),(24)$ линейно независимы с (25). Выражая $\tau, u_{1}, \ldots, u_{3 k-1}$ через $z_{i, t_{1}}, z_{i, t_{2}}, z_{i, t_{3}}$, $i=1, \ldots, k$, из $(27)$ и подставляя результат в $n+1-2 k$ уравнений $(23),(24)$, мы получим систему из $n+1-2 k$ уравнений относительно $n+1-2 k$ неизвестных $z_{1}, \ldots, z_{k}, u_{3 k}, \ldots, u_{n}$. Это трехмерная квазилинейная система второго порядка относительно $z_{i}$ и первого порядка относительно $u_{j}$, коэффициенты которой зависят от $z_{i, t_{1}}, z_{i, t_{2}}, z_{i, t_{3}}, i=1, \ldots, k$, и $u_{3 k}, \ldots, u_{n}$. Ясно, что ее общее решение может быть локально параметризовано с помощью $n+1-k$ функций от двух аргументов. 
В случае $2 k \leqslant n+1<3 k$ функции $z_{i, t_{1}}, z_{i, t_{2}}, z_{i, t_{3}}, i=1, \ldots, k$, функционально зависимы. Мы имеем $3 k-n-1$ уравнений вида

$$
R_{i}\left(z_{1, t_{1}}, z_{1, t_{2}}, z_{1, t_{3}}, \ldots, z_{k, t_{1}}, z_{k, t_{2}}, z_{k, t_{3}}\right)=0, \quad i=1, \ldots, 3 k-n-1,
$$

и $n+1-2 k$ квазилинейных уравнений второго порядка. Всего имеется $(3 k-n-1)+$ $(n+1-2 k)=k$ уравнений относительно $k$ неизвестных функций $z_{1}, \ldots, z_{k}$. Ясно, что ее общее решение локально параметризуется с помощью $n+1-k$ функций от двух аргументов.

Предположим, что $n+1<2 k$. Тогда имеем $n+1+k<3 k$, и это означает, что $3 k$ уравнений $(25)$ линейно зависимы. По-видимому, в этом случае общее решение тоже может быть локально параметризовано с помощью $n+1-k$ функций двух переменных.

Одним из наиболее интересных случаев является $n+1=3 k$, когда мы имеем систему $k$ квазилинейных уравнений второго порядка относительно функций $z_{1}, \ldots, z_{k}$. В простейшем случае $k=2$.

\section{5. ИНТЕГРИРУЕМЫЕ $(1+1)$-МЕРНЫЕ СИСТЕМЫ ГИДРОДИНАМИЧЕСКОГО ТИПА И ГИДРОДИНАМИЧЕСКИЕ РЕДУКЦИИ}

В этом разделе мы приводим интегрируемые $(1+1)$-мерные системы гидродинамического типа (9), описанные в терминах эллиптико-гипергеометрических функций. Эти системы возникают как так называемые гидродинамические редукции наших эллиптических псевдопотенциалов $A_{n, k}$. Результаты и формулы этого раздела похожи на соответствующие результаты и формулы из работы [1].

Под интегрируемостью системы (9) мы понимаем существование у нее бесконечного числа гидродинамических коммутирующих потоков и законов сохранения. Известно [13], что это условие эквивалентно следующим соотношениям для скоростей $v^{i}\left(r^{1}, \ldots, r^{N}\right)$ :

$$
\partial_{j} \frac{\partial_{i} v^{k}}{v^{i}-v^{k}}=\partial_{i} \frac{\partial_{j} v^{k}}{v^{j}-v^{k}}, \quad i \neq j \neq k
$$

Здесь $\partial_{\alpha}=\partial / \partial r^{\alpha}, \alpha=1, \ldots, N$. Если выполнены условия (28), система (9) называется полугамильтоновой.

Основным геометрическим объектом, связанным с полугамильтоновой системой (9), является диагональная метрика $g_{k k}, k=1, \ldots, N$, где

$$
\frac{1}{2} \partial_{i} \ln g_{k k}=\frac{\partial_{i} v^{k}}{v^{i}-v^{k}}, \quad i \neq k
$$

В силу (28) переопределенная система (29) совместна, и функция $g_{k k}$ определяется с точностью до произвольного множителя $\eta_{k}\left(r^{k}\right)$. Метрика $g_{k k}$ называется метрикой, ассочиированной с системой (9). Известно, что две системы гидродинамического типа совместны, если и только если они обладают общей ассоциированной метрикой [13]. 
Рассмотрим систему (11)-(13). Легко проверить, что система (11), (12) находится в инволюции, и поэтому ее общее решение допускает локальную параметризацию с помощью $2 N$ функций одной переменной. При фиксированном решении $\xi_{i}, \tau$ системы (11), (12) система (13) находится в инволюции и, следовательно, имеет однопараметрическое семейство решений.

Пусть $\xi_{1}\left(r^{1}, \ldots, r^{N}\right), \ldots, \quad \xi_{N}\left(r^{1}, \ldots, r^{N}\right), \quad \tau\left(r^{1}, \ldots, r^{N}\right), \quad u_{1}\left(r^{1}, \ldots, r^{N}\right), \ldots$ $\ldots, u_{n}\left(r^{1}, \ldots, r^{N}\right)$ - решение системы (11)-(13). Рассмотрим следующую систему гидродинамического типа:

$$
r_{t}^{i}=\frac{S_{n, k}\left(g_{1}, \xi_{i}\right)}{S_{n, k}\left(g_{2}, \xi_{i}\right)} r_{x}^{i}
$$

где $g_{1}, g_{2}$ - линейно независимые решения системы (4), многочлены $S_{n, k}, k>0$, определены формулой (21), а $S_{n, 0}=S_{n}$ (см. (8)).

ТЕОРема 3. Система (30) является полугамильтоновой. Ее ассоциированная метрика задается формулой

$$
g_{i i}=\left(S_{n, k}\left(g_{2}, \xi_{i}\right) e^{2 \pi i r\left(\tau-\xi_{i}\right)} \frac{\theta^{\prime}(0)^{-s_{1}-\cdots-s_{n}} \theta\left(u_{1}\right)^{s_{1}} \ldots \theta\left(u_{n}\right)^{s_{n}}}{\theta\left(\xi_{i}\right)^{-s_{1}-\cdots-s_{n}} \theta\left(\xi_{i}-u_{1}\right)^{s_{1}} \ldots \theta\left(\xi_{i}-u_{n}\right)^{s_{n}}}\right)^{2} \partial_{i} \tau .
$$

ЗАмечАниЕ 6. Диагональная метрика $g_{i i}$ называется метрикой егоровского muna, если существует потенциал $G$ такой, что $g_{i i}=\partial_{i} G$ для всех $i$. Метрики из теоремы 3, вообще говоря, не являются метриками егоровского типа. Однако при очень специальных значениях параметров $s_{i}$ в $(4)$ существует решение $g_{2} \in \mathcal{H}$ такое, что метрика является егоровской для любого решения системы (11)-(13). Егоровские метрики играют важную роль в теории ВДВВ-уравнений ассоциативности и в теории фробениусовых многообразий [7], [16].

ПреДЛОЖенИЕ 5. Предположим, что решение $\xi_{1}, \ldots, \xi_{N}, \tau, u_{1}, \ldots, u_{n}$ системы (11)-(13) фиксировано. Тогда системы гидродинамического типа

$$
r_{t_{1}}^{i}=\frac{S_{n, k}\left(g_{1}, \xi_{i}\right)}{S_{n, k}\left(g_{3}, \xi_{i}\right)} r_{x}^{i}, \quad r_{t_{2}}^{i}=\frac{S_{n, k}\left(g_{2}, \xi_{i}\right)}{S_{n, k}\left(g_{3}, \xi_{i}\right)} r_{x}^{i}
$$

совместны для любых $g_{1}, g_{2}$.

ЗАмечАниЕ 7. Некоторые совместные системы вида (31) можно также строить, используя предложение 3 . Положим в $(31) g_{2}=Z\left(u_{1}, \ldots, u_{n}, u_{n+1}, \tau\right)$. Здесь $u_{n+1}-$ произвольное решение системы (13) (в которой $n$ заменено на $n+1$ ), отличное от $u_{1}, \ldots, u_{n}$. Ясно, что уравнения $(31)$ совместны для такого $g_{2}$ и любого $g_{1} \in \mathcal{H}$. Более того, из предложения 3 следует, что уравнения (31) совместны, если положить $g_{1}=$ $Z\left(u_{1}, \ldots, u_{n}, u_{n+1}, \tau\right), g_{2}=Z\left(u_{1}, \ldots, u_{n}, u_{n+2}, \tau\right)$ для произвольных решений $u_{n+1}$, $u_{n+2}$ системы (13).

Все члены иерархии, построенной в предложении 5 , допускают бездисперсионное представление Лакса вида

$$
L_{t}=\{L, A\},
$$

2 Теоретическая и математическая физика, т. 161, № 1, 2009 г. 
где $\{L, A\}=A_{p} L_{x}-A_{x} L_{p}$, с одним и тем же $L=L\left(p, r^{1}, \ldots, r^{N}\right)$. А именно, определим функцию $L\left(\xi, r^{1}, \ldots, r^{N}\right)$ с помощью следующей системы:

$$
\partial_{\alpha} L=-\frac{1}{2 \pi i}\left(\rho\left(\xi_{\alpha}-\xi\right)-\rho\left(\xi_{\alpha}\right)\right) L_{\xi} \partial_{\alpha} \tau, \quad \alpha=1, \ldots, N
$$

Легко проверить, что система (33) находится в инволюции, и поэтому функция $L\left(\xi, r^{1}, \ldots, r^{N}\right)$ определена однозначно с точностью до несущественного преобразования $L \rightarrow \lambda(L)$. Для того чтобы найти функцию $L\left(p, r^{1}, \ldots, r^{N}\right)$, нужно выразить $\xi$ через $p$ из (6) для $k=0$ или из $(22)$ для $k>0$.

ПРЕДЛОЖЕНИЕ 6. Пусть $u_{1}, \ldots, u_{n}$ - произвольное решение системь (13). Тогда система (30) допускает бездисперсионное представление Лакса (32), где $A=A_{n, k}$ определен формулой (6) при $k=0$ и бормулой (22) при $k>0$.

Покажем, что интегрируемые двумерные системы (30) определяют гидродинамические редукции для псевдопотенциалов и трехмерных систем из разделов 3 и 4 .

В работах [11], [14] и [19] в терминах гидродинамических редукций было дано определение интегрируемости для систем (1), (2) и (32) соответственно.

Предположим, что существуют пара совместных полугамильтоновых систем гидродинамического типа,

$$
r_{t_{1}}^{i}=v_{1}^{i}\left(r^{1}, \ldots, r^{N}\right) r_{x}^{i}, \quad r_{t_{2}}^{i}=v_{2}^{i}\left(r^{1}, \ldots, r^{N}\right) r_{x}^{i}
$$

и функции $u_{i}=u_{i}\left(r^{1}, \ldots, r^{N}\right)$, удовлетворяющие (1) для любого решения систем (34). Тогда (34) называется гидродинамической редукиией для (1).

ОПРЕДЕЛЕНИЕ 1 [11]. Система вида (1) называется интегрируемой, если уравнение (1) допускает достаточно много гидродинамических редукций для каждого $N \in \mathbb{N}$.

"Достаточно много" означает, что множество гидродинамических редукций может быть локально параметризовано $2 N$ функциями одной переменной. Отметим, что по модулю калибровочных преобразований $r^{i} \rightarrow \lambda_{i}\left(r^{i}\right)$ мы имеем только $N$ существенных функциональных параметров для гидродинамических редукций.

Предположим, что существуют полугамильтонова система гидродинамического типа (9) и функции $u_{i}=u_{i}\left(r^{1}, \ldots, r^{N}\right), L=L\left(p, r^{1}, \ldots, r^{N}\right)$, удовлетворяющие бездисперсионному уравнению Лакса (32) для любого решения $r^{1}(x, t), \ldots, r^{N}(x, t)$ системы (9). Тогда (9) называется гидродинамической редукиией для (32).

ОПРЕДЕЛЕНиЕ 2 [19]. Бездисперсионное уравнение Лакса (32) называется интегрируемым, если оно допускает достаточно много гидродинамических редукций для каждого $N \in \mathbb{N}$. Соответствующий псевдопотенциал $A\left(p, u_{1}, \ldots, u_{n}\right)$ мы также называем интегрируемым. 
ОПРЕДЕЛЕНиЕ 3 [1]. Два интегрируемых псевдопотенциала $A_{1}, A_{2}$ называются согласованными, если система

$$
L_{t_{1}}=\left\{L, A_{1}\right\}, \quad L_{t_{2}}=\left\{L, A_{2}\right\}
$$

допускает достаточно много гидродинамических редукций (34) для каждого $N \in \mathbb{N}$.

Если псевдопотенциалы $A_{1}, A_{2}$ согласованы, то $A=c_{1} A_{1}+c_{2} A_{2}$ интегрируем для любых постоянных $c_{1}, c_{2}$. Действительно, система

$$
r_{t}^{i}=\left(c_{1} v_{1}^{i}(\mathbf{r})+c_{2} v_{2}^{i}(\mathbf{r})\right) r_{x}^{i}
$$

является гидродинамической редукцией для (32).

ОПРЕДЕЛЕНИЕ 4. Под трехмерной системой, ассоииированной с согласованной парой $A_{1}, A_{2}$ мы подразумеваем систему вида (1), эквивалентную условиям совместности для системы

$$
\psi_{t_{2}}=A_{1}\left(\psi_{t_{1}}, u_{1}, \ldots, u_{n}\right), \quad \psi_{t_{3}}=A_{2}\left(\psi_{t_{1}}, u_{1}, \ldots, u_{n}\right) .
$$

Ясно, что система, ассоциированная с парой согласованных псевдопотенциалов, допускает достаточно много гидродинамических редукций и поэтому интегрируема в смысле определения 1.

Следующее утверждение является переформулировкой предложения 6.

Теорема 4. Система (30) является гидродинамической редукиией псевдопотенциала $A_{n, k}$, определенного формулой (6) при $k=0$ и формулой (22) при $k>0$. Напомним, что мы используем обозначения $S_{n} \stackrel{\text { def }}{=} S_{n, 0}, A_{n} \stackrel{\text { def }}{=} A_{n, 0}, P_{n} \stackrel{\text { def }}{=} P_{n, 0}$.

ПреДЛОЖЕНИЕ 7. Предположим, что $g_{1}, g_{2}, g_{3}, h_{1}, \ldots, h_{k} \in \mathcal{H}$ линейно независимы. Определим псевдопотенциаль $A_{1}, A_{2}$ формулами

$$
A_{1}=P_{n, k}\left(g_{1}, \xi\right), \quad A_{2}=P_{n, k}\left(g_{2}, \xi\right), \quad p=P_{n, k}\left(g_{3}, \xi\right) .
$$

Тогда $A_{1}$ и $A_{2}$ согласованъ.

ДокАЗАтельство. Заметим, что система (11)-(13), (33) не содержит $g_{1}, g_{2}, g_{3}$, и поэтому мы имеем семейство функций $L, \xi_{i}, u_{i}, \tau$, задающих гидродинамические редукции вида (30) обоих псевдопотенциалов $A_{1}$ и $A_{2}$. Кроме того, согласно предложению 7 системы

$$
r_{t_{1}}^{i}=\frac{S_{n, k}\left(g_{1}, \xi_{i}\right)}{S_{n, k}\left(g_{3}, \xi_{i}\right)} r_{x}^{i}, \quad r_{t_{2}}^{i}=\frac{S_{n, k}\left(g_{2}, \xi_{i}\right)}{S_{n, k}\left(g_{3}, \xi_{i}\right)} r_{x}^{i}
$$

совместны.

ЗАмЕчАниЕ 8. Из этого результата следует, что трехмерные системы, построенные в разделах 3,4 , допускают достаточно много гидродинамических редукций и поэтому интегрируемы в смысле определения 1.

ЗАмЕЧАниЕ 9. Используя предложение 3, можно построить согласованные псевдопотенциалы $A_{1}$ и $A_{2}$, зависящие от разного числа переменных $u_{i}$. Действительно, пусть $g_{1}, g_{3}, h_{1}, \ldots, h_{k} \in \mathcal{H}$ и $g_{2}=Z\left(u_{1}, \ldots, u_{n}, u_{n+1}, \tau\right)$. Тогда $A_{2}$ зависит от $u_{1}, \ldots, u_{n}, u_{n+1}, \tau$, а $A_{1}$ зависит только от $u_{1}, \ldots, u_{n}, \tau$. 
Благодарности. Авторы благодарны И. М. Кричеверу, М. В. Павлову, В.П. Спиридонову, Б.Л. Фейгину и В.А. Шрамченко за полезные обсуждения. В. В. Соколов был частично поддержан РФФИ (гранты № 08-01-464, 09-01-9244КЭ) и Программой поддержки ведущих научных школ (грант НШ-3472.2008.2).

\section{Список литературы}

[1] A. Odesskii, V. Sokolov, Integrable pseudopotentials related to generalized hypergeometric functions, arxiv:0803.0086.

[2] И. М. Гельфанд, М. И. Граев, В. С. Ретах, УМН, 47:4(286) (1992), 3-82.

[3] V.E. Zakharov, "Dispersionless limit of integrable systems in $2+1$ dimensions", Singular Limits of Dispersive Waves, NATO Adv. Sci. Inst. Ser. B Phys., 320, eds. N. M. Ercolani, I. R. Gabitov, C. D. Levermore, D. Serre, Plenum, New York, 1994, 165-174.

[4] I. M. Krichever, Comm. Math. Phys., 143:2 (1992), 415-429.

[5] В. Е. Захаров, А. Б. Шабат, Функи. анализ и его прил., 13:3 (1979), 13-22.

[6] I. M. Krichever, Comm. Pure Appl. Math., 47:4 (1994), 437-475.

[7] B. A. Dubrovin, "Geometry of 2D topological field theories", Integrable Systems and Quantum Groups, Lecture Notes in Math., 1620, eds. M. Francaviglia, S. Greco, Springer, Berlin, 1996, 120-348.

[8] A. V. Odesskii, Selecta Math., 13:4 (2008), 727-742.

[9] В. П. Спиридонов, УМН, 63:3(381) (2008), 3-72.

[10] J. Gibbons, S. P. Tsarev, Phys. Lett. A, 211:1 (1996), 19-24; 258:4-6 (1999), 263-271.

[11] E. V. Ferapontov, K. R. Khusnutdinova, Comm. Math. Phys., 248:1 (2004), 187-206; J. Phys. A, 37:8 (2004), 2949-2963.

[12] Б. А. Дубровин, С. П. Новиков, УМН, 44:6(270) (1989), 29-98.

[13] С. П. Царев, ДАН СССР, 282:3 (1985), 534-537; С. П. Царев, Изв. АН СССР. Сер. матем., 54:5 (1990), 1048-1068.

[14] M. V. Pavlov, Comm. Math. Phys., 272:2 (2007), 469-505.

[15] V. A. Shramchenko, J. Phys. A, 36:42 (2003), 10585-10605; arxiv: math-ph/0402014.

[16] А. А. Ахметшин, Ю. С. Вольвовский, И. М. Кричевер, УМН, 54:2(326) (1999), 167-168.

[17] E. V. Ferapontov, D. G. Marshal, Math. Ann., 339:1 (2007), 61-99.

[18] M. V. Pavlov, J. Phys. A, 39:34 (2006), 10803-10819.

[19] А. В. Одесский, М. В. Павлов, В. В. Соколов, ТМФ, 154:2 (2008), 249-260. 